\title{
Preparation and Study of Electromagnetic Interference Shielding Materials Comprised of Ni-Co Coated on Web-Like Biocarbon Nanofibers via Electroless Deposition
}

\author{
Xiaohu Huang, Bo Dai, Yong Ren, Jing Xu, and Pei Zhu \\ State Key Laboratory Cultivation Base for Nonmetal Composites and Functional Materials, \\ Southwest University of Science and Technology, Mianyang 621010, China
}

Correspondence should be addressed to Bo Dai; bodai31@vip.sina.com

Received 22 September 2014; Revised 11 December 2014; Accepted 11 December 2014

Academic Editor: Myoung-Woon Moon

Copyright (C) 2015 Xiaohu Huang et al. This is an open access article distributed under the Creative Commons Attribution License, which permits unrestricted use, distribution, and reproduction in any medium, provided the original work is properly cited.

\begin{abstract}
Electromagnetic interference (EMI) shielding materials made of Ni-Co coated on web-like biocarbon nanofibers were successfully prepared by electroless plating. Biocarbon nanofibers (CF) with a novel web-like structure comprised of entangled and interconnected carbon nanoribbons were obtained using bacterial cellulose pyrolyzed at $1200^{\circ} \mathrm{C}$. Paraffin wax matrix composites filled with different loadings $(10,20$, and $30 \mathrm{wt} \%$, resp.) of CF and Ni-Co coated CF (NCCF) were prepared. The electrical conductivities and electromagnetic parameters of the composites were investigated by the four-probe method and vector network analysis. From these results, the EMI shielding efficiencies (SE) of NCCF composites were shown to be significantly higher than that of CF at the same mass fraction. The paraffin wax composites containing $30 \mathrm{wt} \%$ NCCF showed the highest EMI SE of $41.2 \mathrm{~dB}$ (99.99\% attenuation), which are attributed to the higher electrical conductivity and permittivity of the NCCF composites than the CF composites. Additionally, EMI SE increased with an increase in CF and NCCF loading and the absorption was determined to be the primary factor governing EMI shielding. This study conclusively reveals that NCCF composites have potential applications as EMI shielding materials.
\end{abstract}

\section{Introduction}

In recent years electromagnetic interference (EMI) has become a critical problem for electric devices because it may lead to malfunctions such as in airplane control panels, mobile phones, and many computers. In order to address these problems the development of EMI shielding materials received increasing attention [1-3]. Carbon composites have outstanding potential for use in EMI shielding [4-9] due to their excellent electrical properties, low density, high aspect ratio, and high strength and modulus. However the electrical conductivity of carbon materials is considerably lower than that of metals and therefore a greater amount of carbon material is needed to achieve the same shielding effect. Although satisfactory shielding capabilities can be obtained by simply adding more carbon materials, it is difficult to produce composites with high carbon volume fractions when using extrusion or injection molding. In addition, a high carbon volume fraction increases the composites cost and thus limits its commercial use. To optimize the conductivity and EMI shielding effectiveness (SE) of composites, it is necessary to attach metal particles to the surface of carbon materials [10-12]. The introduction of metal particles onto carbon materials has previously been shown to lead to good wettability between the fillers and matrices [13], which implies that the dispersion of carbon materials can improve in the matrices. Amongst the common metal materials, nickel and cobalt are suitable for EMI shielding because these metals exhibit good conductivity and magnetic properties, as well as antioxidant properties. Such good conductivity and magnetic materials can produce a large induced current under perturbation by electromagnetic waves, and, according to Lenz's law, these currents will be effective at weakening the penetration of electromagnetic waves [14]. Therefore, it is expected that carbon materials coated with a layer of metal will have the advantages of good conductivity, magnetic, high intensity, and low density and they combine the properties of carbon materials and the magnetic metal together. Although 
a variety of techniques are available for altering the chemical and physical properties of the surface of carbon materials, electroless plating has been found to be the most suitable method because of its ability to provide a uniform surface and the cost effectiveness of the process [15-17]. Thus, electroless plating is a convenient approach to produce $\mathrm{Ni}$-Co coated carbon materials. However, the application of traditional metal-coated carbon materials for use as EMI shielding composites is hindered by a major obstacle: the cost and limited supply of carbon materials.

Recently, we developed a novel method of fabricating biocarbon nanofiber (CF) with a web-like structure consisting of interconnected carbon nanoribbons using low-cost natural materials such as bacterial cellulose as carbonaceous sources. In this work, we report the preparation process of $\mathrm{CF}$, and the electroless plating method was applied to deposit a layer of Ni-Co coating on the surface of CF. The microstructure, magnetic properties, electrical conductivity, electromagnetic parameters, and EMI SE of the composites were investigated.

\section{Experimental Procedures}

2.1. Preparation of CF. Web-like structure CF was obtained by heat treatment of bacterial cellulose in a high-temperature tube furnace for $4 \mathrm{~h}$ under a nitrogen atmosphere at $1200^{\circ} \mathrm{C}$. Afterwards, dried CF was dispersed in absolute ethanol and mechanically milled into a slurry. The resulting CF was dried using a vacuum at $60^{\circ} \mathrm{C}$. The bacterial cellulose was kindly provided by Hainan Ye Guo Foods Co., Ltd., Hainan, China.

2.2. Pretreatments and Electroless Plating of $\mathrm{CF}$ with $\mathrm{Ni}$ Co. For metal-coated CF/polymer matrix composites, the most important factor affecting the SE of composites is the adhesion between the metal coating and CF. Good adhesion can reduce the delamination of metal deposits by shear stress during compounding and can maintain the conductive network. In order to enhance the interfacial adhesion between the CF and the coating, CF was subjected to an oxidation treatment before surface activation. The CF was immersed in an aqueous solution of $200 \mathrm{~g} / \mathrm{L}\left(\mathrm{NH}_{4}\right)_{2} \mathrm{~S}_{2} \mathrm{O}_{8}$ and $100 \mathrm{~mL} / \mathrm{L}$ of $\mathrm{H}_{2} \mathrm{SO}_{4}$ (the acid solution readily etches the surface of $\mathrm{CF}$ ) by sonication at $35^{\circ} \mathrm{C}$ for $80 \mathrm{~min}$, then was filtered, and thoroughly washed with distilled water until the washings reached $\mathrm{pH}=7$. This procedure promotes the coarsening of the carbon fiber, which can form different functional groups on the CF surface. These surface functional groups can form anchoring sites for metallic nanoparticles, thereby improving the adhesion between the CF and the nanoparticles [18]. The coating procedure consisted of three stages: sensitization, activation, and metallization. The $\mathrm{CF}$ and the optimum operating conditions of the sensitized, activated, and plating solutions were listed in Table 1. Firstly, coarsened CF was sensitized for $30 \mathrm{~min}$ at $25^{\circ} \mathrm{C}$ to adsorb $\mathrm{Sn}^{2+}$ ions. The $\mathrm{CF}$ was then filtered and washed with distilled water, and the sensitized CF was placed in an activated solution at $25^{\circ} \mathrm{C}$ for 30 min to adsorb Pd nuclei on the CF surface. Sonication was applied during the sensitization and activation treatment to promote uniform coating of the surface of CF. Finally, the
TABLE 1: Composition and operating conditions for the different stages in nickel-cobalt electroless coating.

\begin{tabular}{|c|c|}
\hline Stage & Solution \\
\hline Sensitization & $\begin{array}{c}23 \mathrm{~g} / \mathrm{L} \mathrm{SnCl} \cdot 2 \mathrm{H}_{2} \mathrm{O} \\
10 \mathrm{~mL} / \mathrm{L} \mathrm{HCl}\end{array}$ \\
\hline Activation & $\begin{array}{c}0.25 \mathrm{~g} / \mathrm{L} \mathrm{PdCl} \\
30 \mathrm{~mL} / \mathrm{L} \mathrm{HCl}\end{array}$ \\
\hline Metallization & $\begin{array}{c}17 \mathrm{~g} / \mathrm{L} \mathrm{NiSO}_{4} \cdot 6 \mathrm{H}_{2} \mathrm{O} \\
9 \mathrm{~g} / \mathrm{L} \mathrm{CoSO}_{4} \cdot 7 \mathrm{H}_{2} \mathrm{O} \\
80 \mathrm{~g} / \mathrm{L} \mathrm{Na}_{3} \mathrm{C}_{6} \mathrm{H}_{5} \mathrm{O}_{7} \cdot 2 \mathrm{H}_{2} \mathrm{O} \\
35 \mathrm{~g} / \mathrm{L} \mathrm{Na}_{2} \mathrm{H}_{2} \mathrm{PO}_{2} \cdot \mathrm{H}_{2} \mathrm{O} \\
49 \mathrm{~g} / \mathrm{L}\left(\mathrm{NH}_{4}\right)_{2} \mathrm{SO}_{4}\end{array}$ \\
\hline
\end{tabular}

activated CF was washed with distilled water and then added to a bath for metallization, which was carried out at $35^{\circ} \mathrm{C}$ for 15 min with magnetic stirring. Ammonia solution was used as a buffer to maintain a $\mathrm{pH}$ value of 9. Chemicals used in the experiments were all of analytical grade and were purchased from Kelong Reagent Co. Ltd., Chengdu, China. After plating, the Ni-Co coated CF (NCCF) was washed with distilled water and then heat-treated at $450^{\circ} \mathrm{C}$ in a nitrogen atmosphere for $2 \mathrm{~h}$ in a high-temperature tube furnace. After heat treatment, the electrical conductivity of NCCF will improve due to the phase transformation of NCCF [19].

2.3. Fabrication of Test Samples. CF and NCCF were dispersed in dimethylbenzene with paraffin wax by sonication at $60^{\circ} \mathrm{C}$ for $50 \mathrm{~min}$, respectively. Both CF and NCCF dispersed efficiently in the paraffin wax matrix. Test samples with different weight percentages of CF and NCCF (10,20, and $30 \mathrm{wt} \%$ ) were fabricated according to the above methods. For electrical conductivity characterization, rectangular plates $(10 \mathrm{~mm} \times 10 \mathrm{~mm} \times 1.5 \mathrm{~mm})$ of samples were molded under $7 \mathrm{MPa}$ pressure. For EMI SE characterization, samples were pressed into cylindrical dies with $7.0 \mathrm{~mm}$ outer diameter, $3.0 \mathrm{~mm}$ inner diameter, and approximately $2.5 \mathrm{~mm}$ height at $2 \mathrm{MPa}$.

2.4. Characterization. The morphologies of CF and NCCF were analyzed by transmission electron microscopy (TEM, Libra 200FE, Carl Zeiss SMT Pte Ltd., Germany) coupled with energy dispersive spectroscopy (EDS). Raman spectra were recorded on a Raman spectrometer (Invia, Renishaw, $\mathrm{UK}$ ), with an excitation laser wavelength of $514.5 \mathrm{~nm}$. An Xray diffractometer (XRD, X'pert PRO, PANalytical, Netherlands) equipped with a rotating anode and $\mathrm{Cu} \mathrm{K} \alpha$ radiation $(\lambda=0.15406 \mathrm{~nm})$ over an incident angle $(2 \theta)$ range of $10-80^{\circ}$ was used to identify the crystalline phases of the coating. The static magnetic properties were determined using a vibration sample magnetometer (BKT-4500Z, Beijing Ze Tian Wei Ye Science and Technology Co., Ltd., China). The sheet resistance $\left(R_{s}\right)$ of the samples was measured by the fourprobe method using a Keithley 2400 multimeter (Cleveland, $\mathrm{OH}, \mathrm{USA}$ ). The electromagnetic and scattering parameters of the samples were measured using a vector network analyzer (E5071C, Agilent Science and Technology Co., Ltd., USA) in the $8.2-12.4 \mathrm{GHz}$ (X-band) frequency range. 


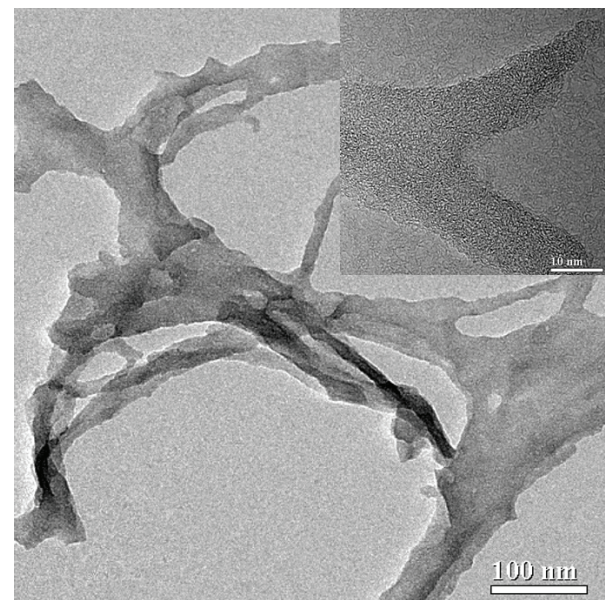

(a)

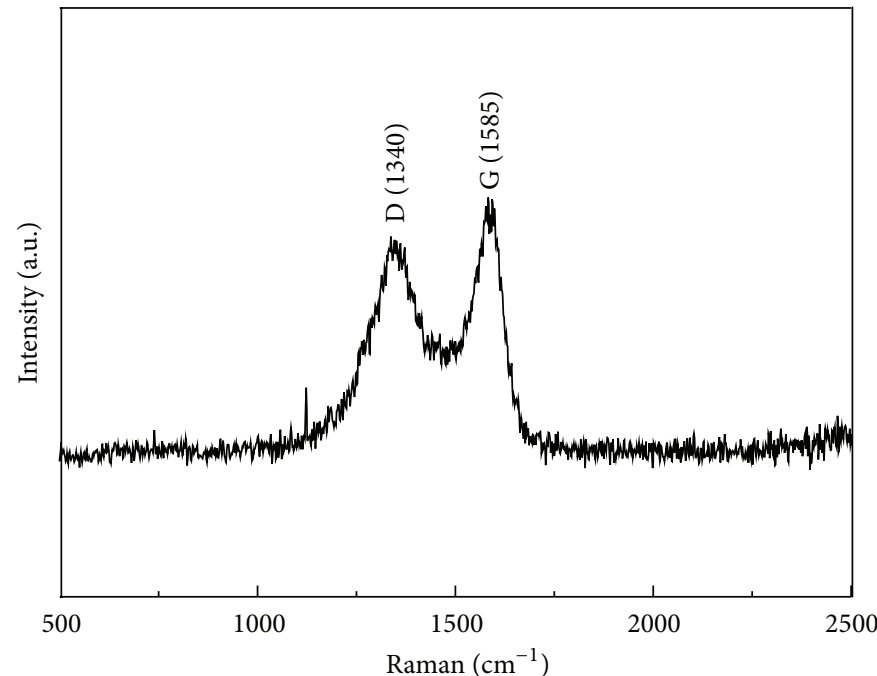

(b)

Figure 1: (a) TEM micrographs of CF and (b) Raman spectra of CF.

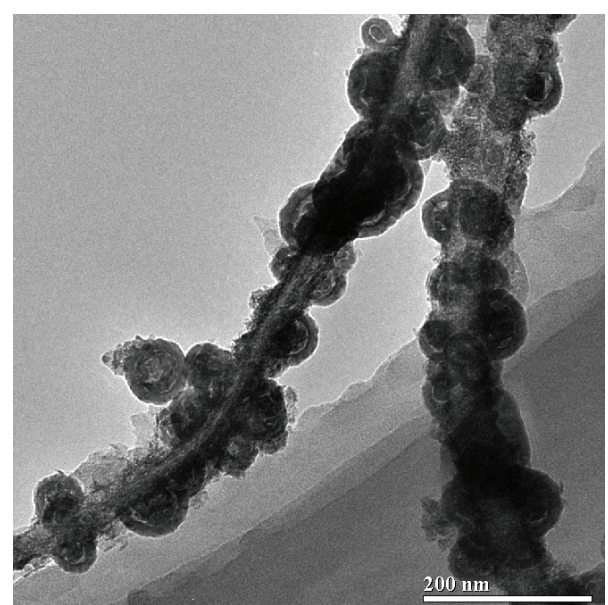

FIgURE 2: TEM micrographs of NCCF.

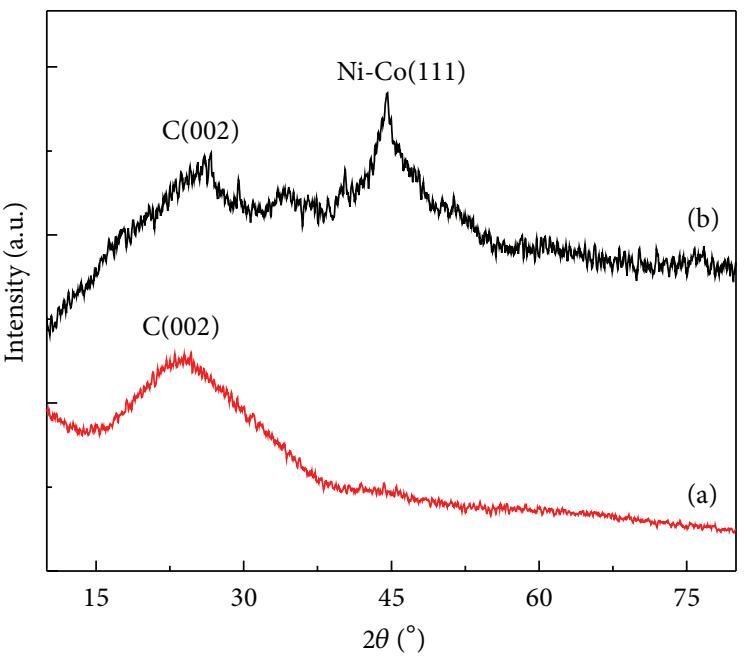

FIGURE 3: XRD patterns of (a) pristine CF and (b) NCCF.

\section{Results and Discussions}

The morphology of nanofibers is an important factor affecting the SE of fiber composites [20]. Figure 1(a) shows the TEM image of the obtained CF, which reveals that the CF are web-like and the diameters can be up to $20 \mathrm{~nm}$. A higher magnification shows that a small amount of graphitic multilayer structure was seen as shown in the inset figure, showing that the CF is slightly graphitized. Figure $1(\mathrm{~b})$ shows the Raman spectra of the obtained CF. In the Raman spectra, two fundamental vibrations are observed at $1340 \mathrm{~cm}^{-1}$ and $1585 \mathrm{~cm}^{-1}$ for CF, which are attributed to the $\mathrm{D}$ and $\mathrm{G}$ bands, respectively. The D peak can be assigned to the disorderactivated Raman mode, while the $G$ peak arises from the $\mathrm{sp}^{2}$ hybridized carbon [21]. Figure 2 is a representative TEM image of NCCF, which clearly indicates that nickel-cobalt spherical grains were formed on the CF surface. The coating layer tended to form as spherical grains, perhaps because the surface exhibits high curvature and on a curved surface the normal growth rate is higher than the lateral growth rate. Obviously the diameter of NCCF is wider than the obtained CF after electroless plating. These results indicate that the wider NCCF in the diameter is highly beneficial to the improvement electrical conductivity of the CF. The EDS spectrum showed that the major components in NCCF are carbon, nickel, cobalt, and oxygen.

The phase structures of both CF and NCCF have been determined by XRD. Figure 3 shows the XRD patterns of (a) the pristine CF and (b) NCCF. It suggests that the CF and NCCF display amorphous structures. In the diffraction pattern (Figure 3(a)) of pristine CF, a broad and weak peak at $2 \theta=24.35^{\circ}$ is observed, which is assigned to the (002) plane of graphitic carbon, which is in good agreement with Figure 1(b). In the case of NCCF, a new broad peak other than the (002) diffraction peak of graphitized CF is clearly 


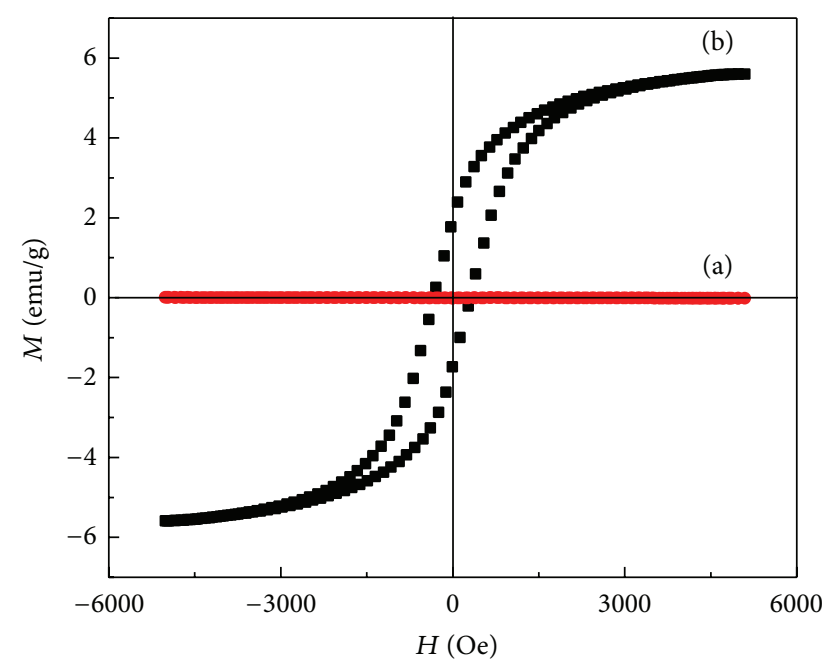

FIgURE 4: Magnetic hysteresis loop of (a) CF and (b) NCCF at $300 \mathrm{~K}$.

observed at around $44.57^{\circ}$ as shown in Figure 3(b), which originates from the deposited Ni-Co coating layer. The results of the XRD analyses indicate that the Ni-Co nanoparticles were successfully deposited on the surface of the CF.

Figure 4 shows the magnetic hysteresis loops of $\mathrm{CF}$ and NCCF. The CF exhibited very little magnetization or coercivity, while the NCCF exhibited obvious magnetization. The saturation magnetization and coercivity of NCCF were $312 \mathrm{Oe}$ and $5.58 \mathrm{emu} / \mathrm{g}$, respectively. The magnetic properties of NCCF are significantly higher than those of CF, which indicates that the $\mathrm{CF}$ has been covered successfully by a layer of ferromagnetic Ni-Co nanoparticles.

Conductivity is another factor affecting the shielding properties of a material [22]. Electrical conductivity is calculated according to the following equation:

$$
\sigma=\frac{1}{R_{s} t},
$$

where $\sigma, R_{s}$, and $t$ represent the direct current conductivity, sheet resistance, and sheet thickness, respectively. The relationship between electrical conductivity and nanofibers (CF and NCCF) loading is illustrated in Figure 5, which shows that the electrical conductivity of NCCF is bigger than that of pure $\mathrm{CF}$ at the same mass fraction. The composites containing $30 \mathrm{wt} \%$ NCCF exhibit the highest electrical conductivity of $1313 \mathrm{~S} / \mathrm{m}$, suggesting that the addition of electrical Ni-Co nanoparticles onto CF is highly beneficial to the electrical conductivity of the composites. The electrical conductivity increases with nanofibers loading because the interfacial affinity between nanofibers and the paraffin wax matrix increases the number of conductive pathways for electron transfer. The excellent electrical conductivity of the composites can improve the electromagnetic energy absorption and dissipation capabilities, resulting in enhancement of microwave shielding effectiveness [23].

To evaluate the EMI performance of CF and the NCCF composites, we thus measured the permittivity $\left(\varepsilon=\varepsilon^{\prime}-j \varepsilon^{\prime \prime}\right.$ constitutes the real part $\left(\varepsilon^{\prime}\right)$ and imaginary part $\left(\varepsilon^{\prime \prime}\right)$, resp.) of our composites in the frequency range of $8.2-12.4 \mathrm{GHz}$.

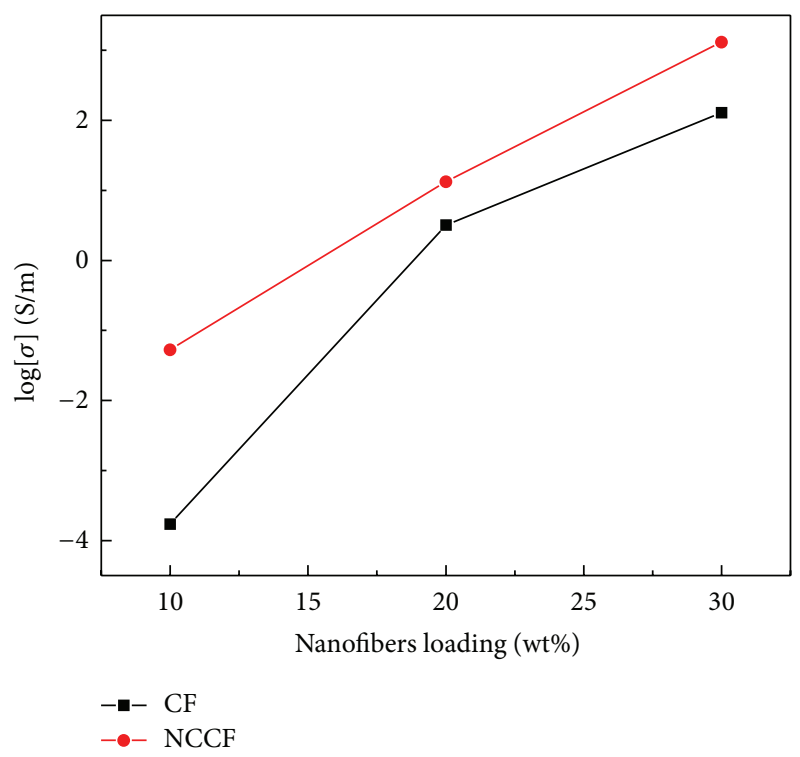

FIgURE 5: Electrical conductivity of CF and NCCF. The thickness of all the samples was $1.5 \mathrm{~mm}$.

Figure 6 shows the room temperature permittivity of the pure $\mathrm{CF}$ and the NCCF composites. The results indicate that the real part and imaginary part of the permittivity of NCCF are bigger than those of pure CF at the same mass fraction. According to the theory of permittivity, when electromagnetic radiation is incident on metallic surface the electric field induces two types of electrical currents within the material (displacement currents and conduction). The former arises from bound charges, that is, polarization effects (real part of permittivity) which mainly involves unpaired point defects, and the latter arises due to free electrons giving rise to electric loss (imaginary part of permittivity) [24]. In other words, the real part of the composites permittivity is a measure of the number of the polarization centers inside the material originating from defects in the nanofibers structure. Therefore, the NCCF nanofibers increase the number of structural defects. The increase of the imaginary part of the permittivity can be attributed to the enhanced electrical conductivity of the composites. Both the real and imaginary parts of permittivity increase as the loading of the $\mathrm{CF}$ or NCCF was increased, which reflects an increase in both the number of polarization centers and dissipating mobile charge carriers, leading to higher permittivity. While this explanation implies that more structural defects and high conductivity result in high permittivity, nevertheless, it is noted that the permittivity decreases with increasing frequency. This behavior is due to the lag of the induced electric field in the composites in response to the reversing external E-field, which leads to a reduction in electronic oscillation at high frequency [25].

The EMI SE of a material is represented by the following equation:

$$
\mathrm{SE}_{T}=10 \log \left(\frac{P_{\text {in }}}{P_{\text {out }}}\right)=\mathrm{SE}_{R}+\mathrm{SE}_{A}+\mathrm{SE}_{M},
$$




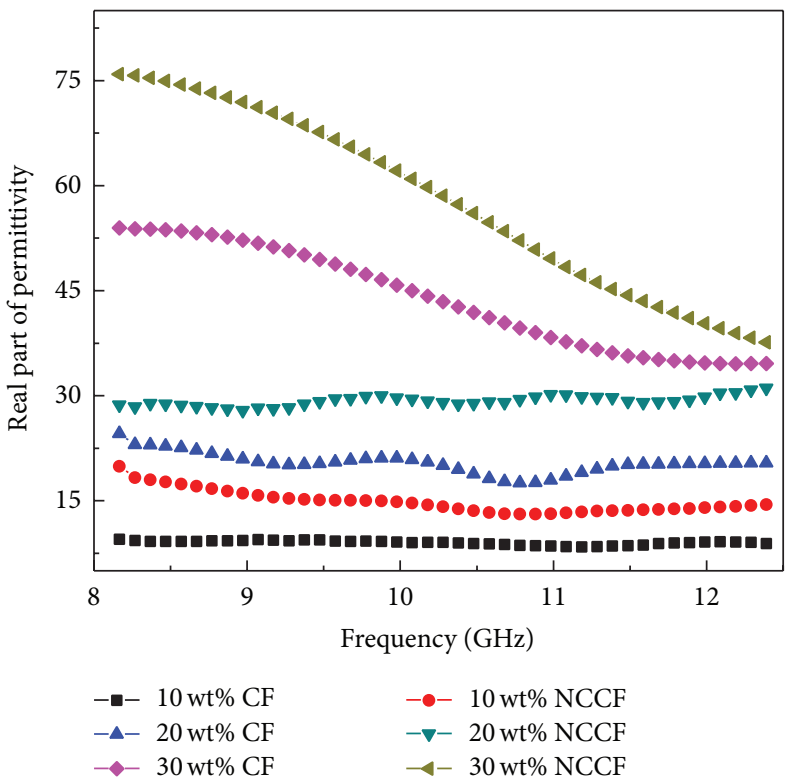

(a)

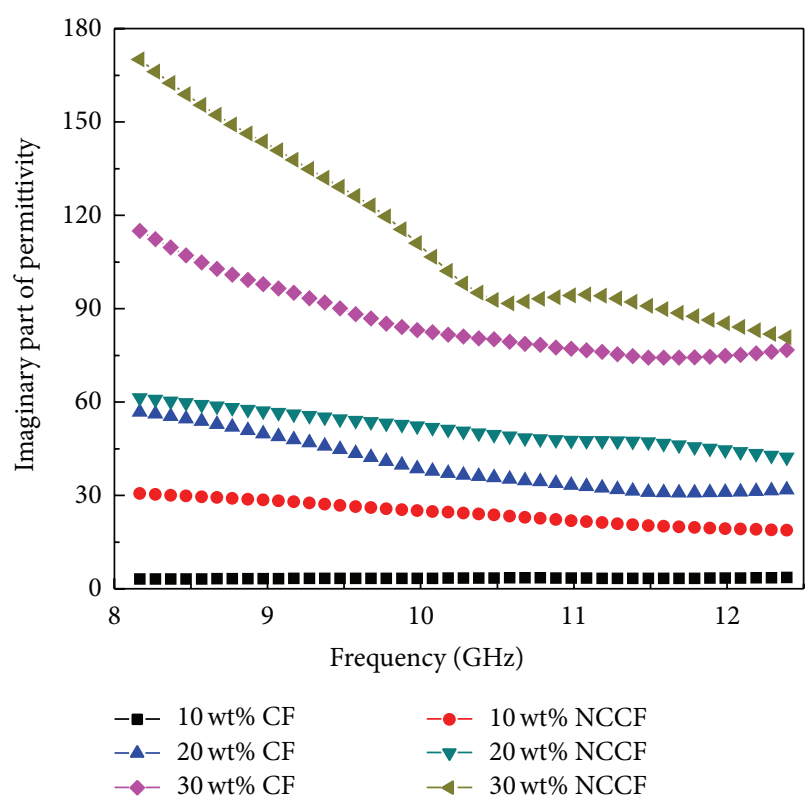

(b)

FIGURE 6: (a) The real and (b) the imaginary parts of the permittivity of the composites as a function of frequency.

where $P_{\text {in }}$ and $P_{\text {out }}$ are the power (electric or magnetic field) of incident and transmitted EM waves, respectively, and $\mathrm{SE}_{R}$, $\mathrm{SE}_{A}$, and $\mathrm{SE}_{M}$ are the $\mathrm{SE}$ due to reflection, absorption, and multiple reflections, respectively [26]. Reflection occurs as a result of mobile charge carriers (electrons or holes) in the shield material which interact with the electromagnetic field in the radiation; the absorption is due to the energy dissipation when electromagnetic wave interacts with electric or magnetic dipoles in the shield material; the multiple reflections refer to the reflection at different surfaces or interfaces in the shield material [27].

$S_{11}$ (or $\left.S_{11}\right)$ and $S_{12}\left(\right.$ or $\left.S_{21}\right)$ are the scattering parameters ( $S$-parameters) of the two-port vector network analyzer system and can be related to reflectance and transmittance as $R=\left|S_{11}\right|^{2}=\left|S_{22}\right|^{2}$ and $T=\left|S_{21}\right|^{2}=\left|S_{12}\right|^{2}$. The absorbance (A) can be written as $A=(1-R-T)$. When $\mathrm{SE}_{A}$ is higher than $10 \mathrm{~dB}, \mathrm{SE}_{M}$ can be ignored [28]. Therefore, $\mathrm{SE}_{T}$ can be conveniently expressed as $\mathrm{SE}_{T}=\mathrm{SE}_{R}+\mathrm{SE}_{A}$. The EMI SE was calculated based on the $S$-parameters as follows:

$$
\begin{gathered}
\mathrm{SE}_{T}=10 \log \frac{1}{\left|S_{12}\right|^{2}}=10 \log \frac{1}{\left|S_{21}\right|^{2}} \\
\mathrm{SE}_{R}=10 \log \left(\frac{1}{1-\left|S_{11}\right|^{2}}\right) \\
\mathrm{SE}_{A}=10 \log \left(\frac{1-\left|S_{11}\right|^{2}}{\left|S_{12}\right|^{2}}\right)
\end{gathered}
$$

where $\left|S_{i j}\right|^{2}$ represents the power transmitted from port $i$ to port $j[29]$.

The influence of mass fraction on the $\mathrm{SE}_{T}$ values of the composites at $10 \mathrm{GHz}$ is shown in Figure 7. The experimental trend is similar to the trend of the electric conductivity of the CF and NCCF composites. In line with the above data concerning conductivity and permittivity, the $\mathrm{SE}_{T}$ values of the CF and NCCF composites increased with the weight percentage. This may be ascribed to the increase of electrical conductivity of the composites. As was observed for the conductivity of composites, the $\mathrm{SE}_{T}$ of the NCCF composite is significantly higher than that of CF at the same mass fraction. The Ni-Co coating on the CF can lead to a decrease in the contact loss between CF due to the formation of metal-metal interfaces. This behavior can improve the electrical conductivity and subsequently the $\mathrm{SE}_{T}$ of the NCCF composites.

To clarify the EMI shielding mechanism of the CF and NCCF composites, the EMI $\mathrm{SE}_{R}, \mathrm{SE}_{A}$, and total $\mathrm{SE}_{T}$ of the composites with $30 \mathrm{wt} \%$ conductive nanofibers in the $8.2-12.4 \mathrm{GHz}$ range are shown in Figure 8. From the figure, $\mathrm{SE}_{A}$ was shown to be ca. 34.3 and $37.3 \mathrm{~dB}$ for $\mathrm{CF}$ and NCCF composites at $12.4 \mathrm{GHz}$, respectively. For the reflection component, $\mathrm{SE}_{R}$ was ca. 2.6 and $3.9 \mathrm{~dB}$ for $\mathrm{CF}$ and NCCF composites at $12.4 \mathrm{GHz}$, respectively. These results demonstrate that the $\mathrm{SE}_{T}$ of the NCCF composites can be up to $41.2 \mathrm{~dB}$ (99.99\% attenuation), which is higher than that of the pristine CF composites [30]. It has been observed that, for conduct CF and NCCF composites, the $\mathrm{SE}_{T}$ is mainly dominated by absorption while the $\mathrm{SE}_{R}$ contributes little.

\section{Conclusions}

In conclusion, NCCF was successfully manufactured by electroless plating. This work demonstrates that both the electrical conductivity and the EMI SE increase with nanofibers loading and the paraffin wax composite containing $30 \mathrm{wt} \%$ NCCF exhibited the highest electrical conductivity of 


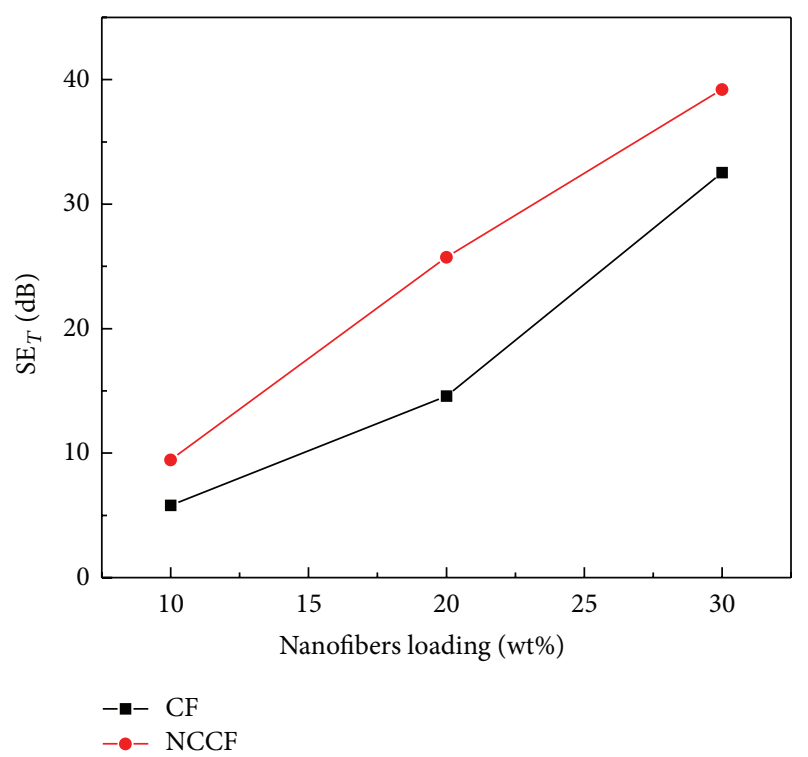

Figure 7: EMI shielding effectiveness of CF and NCCF composites at $10 \mathrm{GHz}$. The thickness of all the samples was $2.5 \mathrm{~mm}$.

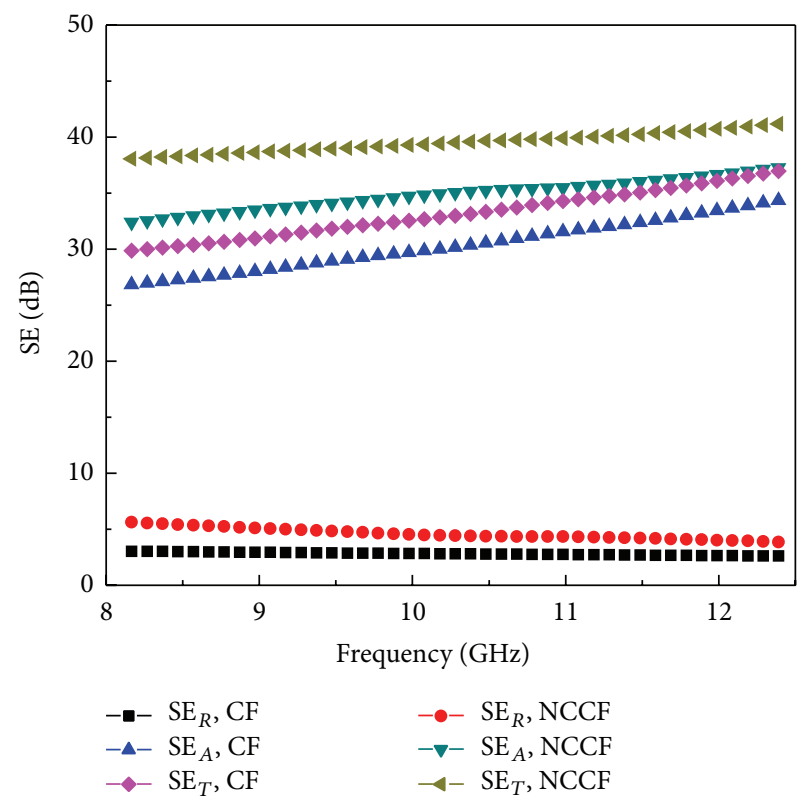

FIGURE 8: EMI shielding effectiveness of CF and NCCF composites with $30 \mathrm{wt} \%$ conductive nanofibers as a function of frequency. The thickness of all the samples was $2.5 \mathrm{~mm}$.

$1313 \mathrm{~S} / \mathrm{m}$ and EMI SE value of $41.2 \mathrm{~dB}$ (99.99\% attenuation). This result may be attributed to the presence of $\mathrm{Ni}$-Co coated on the CF, which can lead to decrease in the contact loss between CF due to the formation of metal-metal interfaces. This behavior can improve the electrical conductivity and permittivity and subsequently improve the EMI SE. The SE of composites increases with nanofibers loading and the absorption is the primary factor governing EMI shielding. This study reveals that the NCCF composites have potential applications as EMI shielding materials.

\section{Conflict of Interests}

The authors declare that there is no conflict of interests regarding the publication of this paper.

\section{Acknowledgment}

This work was supported by the Program for New Century Excellent Talents in university (no. MCET-11-1061).

\section{References}

[1] N. Joseph and M. T. Sebastian, "Electromagnetic interference shielding nature of PVDF-carbonyl iron composites," Materials Letters, vol. 90, pp. 64-67, 2013.

[2] S. X. Jiang and R. H. Guo, "Electromagnetic shielding and corrosion resistance of electroless $\mathrm{Ni}-\mathrm{P} / \mathrm{Cu}-\mathrm{Ni}$ multilayer plated polyester fabric," Surface and Coatings Technology, vol. 205, no. 17-18, pp. 4274-4279, 2011.

[3] M. H. Al-Saleh, G. A. Gelves, and U. Sundararaj, "Copper nanowire/polystyrene nanocomposites: lower percolation threshold and higher EMI shielding," Composites Part A: Applied Science and Manufacturing, vol. 42, no. 1, pp. 92-97, 2011.

[4] G.-H. Kang and S.-H. Kim, "Electromagnetic wave shielding effectiveness based on carbon microcoil-polyurethane composites," Journal of Nanomaterials, vol. 2014, Article ID 727024, 6 pages, 2014.

[5] W.-L. Song, M.-S. Cao, M.-M. Lu et al., "Flexible graphene/ polymer composite films in sandwich structures for effective electromagnetic interference shielding," Carbon, vol. 66, pp. 6776, 2014.

[6] A. Gupta and V. Choudhary, "Electrical conductivity and shielding effectiveness of poly(trimethylene terephthalate)/ multiwalled carbon nanotube composites," Journal of Materials Science, vol. 46, no. 19, pp. 6416-6423, 2011.

[7] D.-X. Yan, H. Pang, L. Xu et al., "Electromagnetic interference shielding of segregated polymer composite with an ultralow loading of in situ thermally reduced graphene oxide," Nanotechnology, vol. 25, no. 14, Article ID 145705, 2014.

[8] A. P. Singh, B. K. Gupta, M. Mishra, A. Chandra, R. B. Mathur, and S. K. Dhawan, "Multiwalled carbon nanotube/cement composites with exceptional electromagnetic interference shielding properties," Carbon, vol. 56, pp. 86-96, 2013.

[9] D. D. L. Chung, "Carbon materials for structural self-sensing, electromagnetic shielding and thermal interfacing," Carbon, vol. 50, no. 9, pp. 3342-3353, 2012.

[10] B.-J. Kim, K.-M. Bae, Y. S. Lee, K.-H. An, and S.-J. Park, "EMI shielding behaviors of Ni-coated MWCNTs-filled epoxy matrix nanocomposites," Surface and Coatings Technology, vol. 242, pp. 125-131, 2014.

[11] R. R. Bonaldi, E. Siores, and T. Shah, "Characterization of electromagnetic shielding fabrics obtained from carbon nanotube composite coatings," Synthetic Metals, vol. 187, no. 1, pp. 1-8, 2014.

[12] B.-J. Kim, W.-K. Choi, M.-K. Um, and S.-J. Park, "Effects of nickel coating thickness on electric properties of nickel/carbon hybrid fibers," Surface and Coatings Technology, vol. 205, no. 11, pp. 3416-3421, 2011.

[13] B.-J. Kim, K.-M. Bae, M.-K. Seo, K.-H. An, and S.-J. Park, "Roles of Ni/CNTs hybridization on rheological and mechanical 
properties of CNTs/epoxy nanocomposites," Materials Science and Engineering: A, vol. 528, no. 15, pp. 4953-4957, 2011.

[14] K. Ji, H. Zhao, J. Zhang, J. Chen, and Z. Dai, "Fabrication and electromagnetic interference shielding performance of opencell foam of a $\mathrm{Cu}-\mathrm{Ni}$ alloy integrated with CNTs," Applied Surface Science, vol. 311, pp. 351-356, 2014.

[15] H. Bi, K.-C. Kou, K. K. Ostrikov, L.-K. Yan, and Z.-C. Wang, "Microstructure and electromagnetic characteristics of Ni nanoparticle film coated carbon microcoils," Journal of Alloys and Compounds, vol. 478, no. 1-2, pp. 796-800, 2009.

[16] F. Wang, S. Arai, and M. Endo, "Metallization of multi-walled carbon nanotubes with copper by an electroless deposition process," Electrochemistry Communications, vol. 6, no. 10, pp. 1042-1044, 2004.

[17] T. Homma, Y. Sezai, and T. Osaka, "A study on growth processes of CoNiP perpendicular magnetic anisotropy films electrolessdeposited at room temperature," Electrochimica Acta, vol. 42, no. 20-22, pp. 3041-3047, 1997.

[18] Y. Li, C. Zhu, and C. Wang, "Controllable synthesis, characterization and microwave absorption properties of magnetic $\mathrm{Ni}_{1-x} \mathrm{Co}_{x} \mathrm{P}$ alloy nanoparticles attached on carbon nanotubes," Journal of Physics D: Applied Physics, vol. 41, no. 12, Article ID 125303, 2008.

[19] C.-Y. Huang, W.-W. Mo, and M.-L. Roan, "The influence of heat treatment on electroless-nickel coated fibre (ENCF) on the mechanical properties and EMI shielding of ENCF reinforced ABS polymeric composites," Surface and Coatings Technology, vol. 184, no. 2-3, pp. 123-132, 2004.

[20] W.-S. Jou, H.-Z. Cheng, and C.-F. Hsu, “The electromagnetic shielding effectiveness of carbon nanotubes polymer composites," Journal of Alloys and Compounds, vol. 434-435, pp. 641645, 2007.

[21] D. C. Elias, R. R. Nair, T. M. G. Mohiuddin et al., "Control of graphene's properties by reversible hydrogenation: evidence for graphane," Science, vol. 323, no. 5914, pp. 610-613, 2009.

[22] N. Li, Y. Huang, F. Du et al., "Electromagnetic Interference (EMI) shielding of single-walled carbon nanotube epoxy composites," Nano Letters, vol. 6, no. 6, pp. 1141-1145, 2006.

[23] B. P. Singh, V. Choudhary, P. Saini, and R. B. Mathur, "Designing of epoxy composites reinforced with carbon nanotubes grown carbon fiber fabric for improved electromagnetic interference shielding," AIP Advances, vol. 2, no. 2, Article ID 022151, 2012.

[24] S.-L. Shi and J. Liang, "The effect of multi-wall carbon nanotubes on electromagnetic interference shielding of ceramic composites," Nanotechnology, vol. 19, no. 25, Article ID 255707, 2008.

[25] C. Wang, X. J. Han, P. Xu, L. Zhang, Y. C. Du, and S. R. Hu, “The electromagnetic property of chemically reduced graphene oxide and its application as microwave absorbing material," Applied Physics Letters, vol. 98, no. 7, Article ID 072906, 2011.

[26] A. P. Singh, M. Mishra, A. Chandra, and S. K. Dhawan, "Graphene oxide/ferrofluid/cement composites for electromagnetic interference shielding application," Nanotechnology, vol. 22, no. 46, Article ID 465701, 2011.

[27] T. K. Gupta, B. P. Singh, R. B. Mathur, and S. R. Dhakate, "Multi-walled carbon nanotube-graphene-polyaniline multiphase nanocomposite with superior electromagnetic shielding effectiveness," Nanoscale, vol. 6, no. 2, pp. 842-851, 2014.

[28] Y. Chen, Y. Li, M. Yip, and N. Tai, "Electromagnetic interference shielding efficiency of polyaniline composites filled with graphene decorated with metallic nanoparticles," Composites Science and Technology, vol. 80, pp. 80-86, 2013.
[29] M. H. Al-Saleh, W. H. Saadeh, and U. Sundararaj, "EMI shielding effectiveness of carbon based nanostructured polymeric materials: a comparative study," Carbon, vol. 60, pp. 146-156, 2013.

[30] B. Dai, Y. Ren, G. Wang, Y. Ma, P. Zhu, and S. Li, "Microstructure and dielectric properties of biocarbon nanofiber composites," Nanoscale Research Letters, vol. 8, no. 1, pp. 1-6, 2013. 

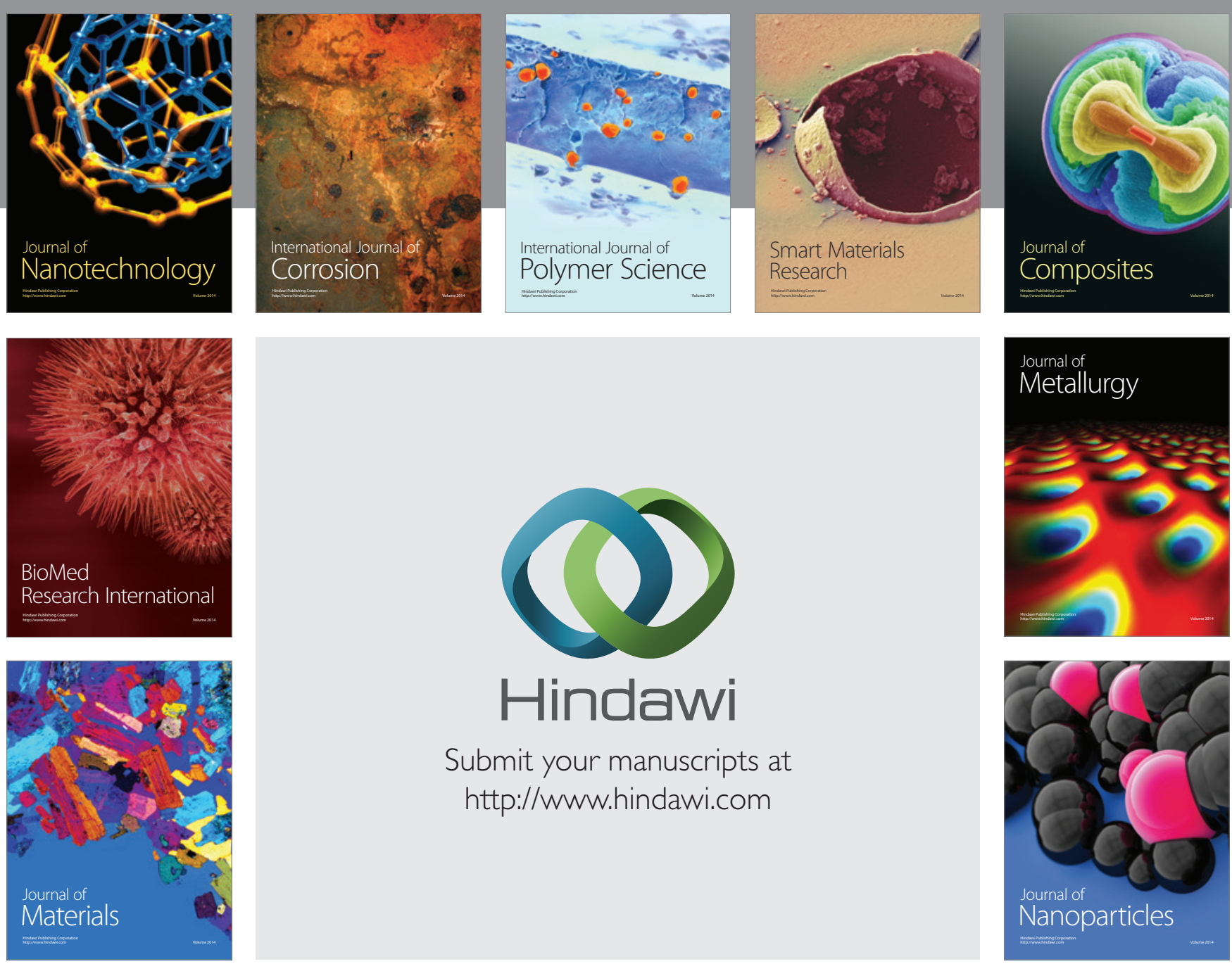

Submit your manuscripts at http://www.hindawi.com
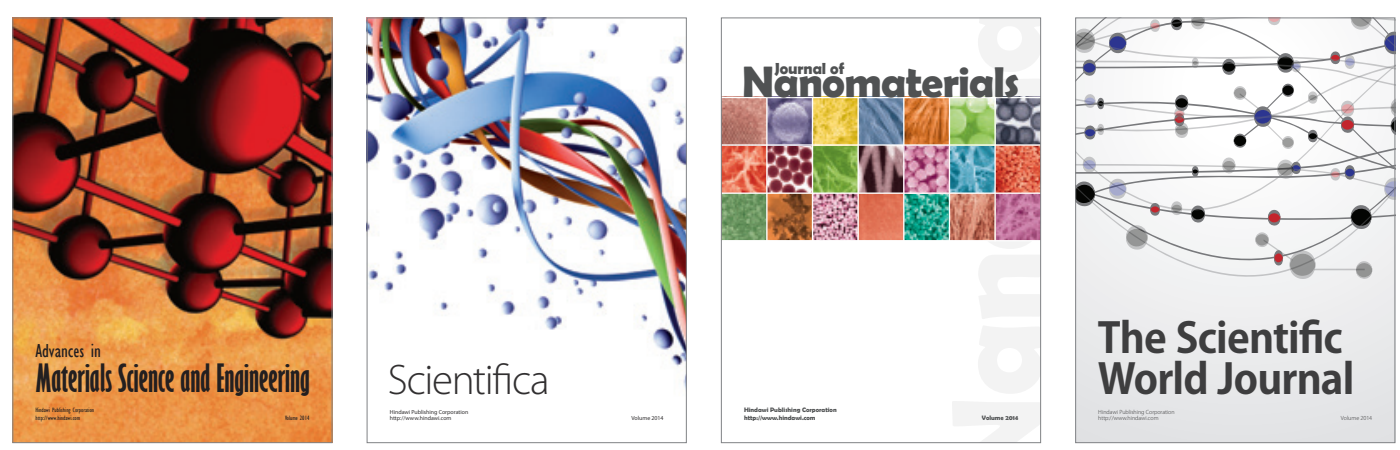

\section{The Scientific World Journal}
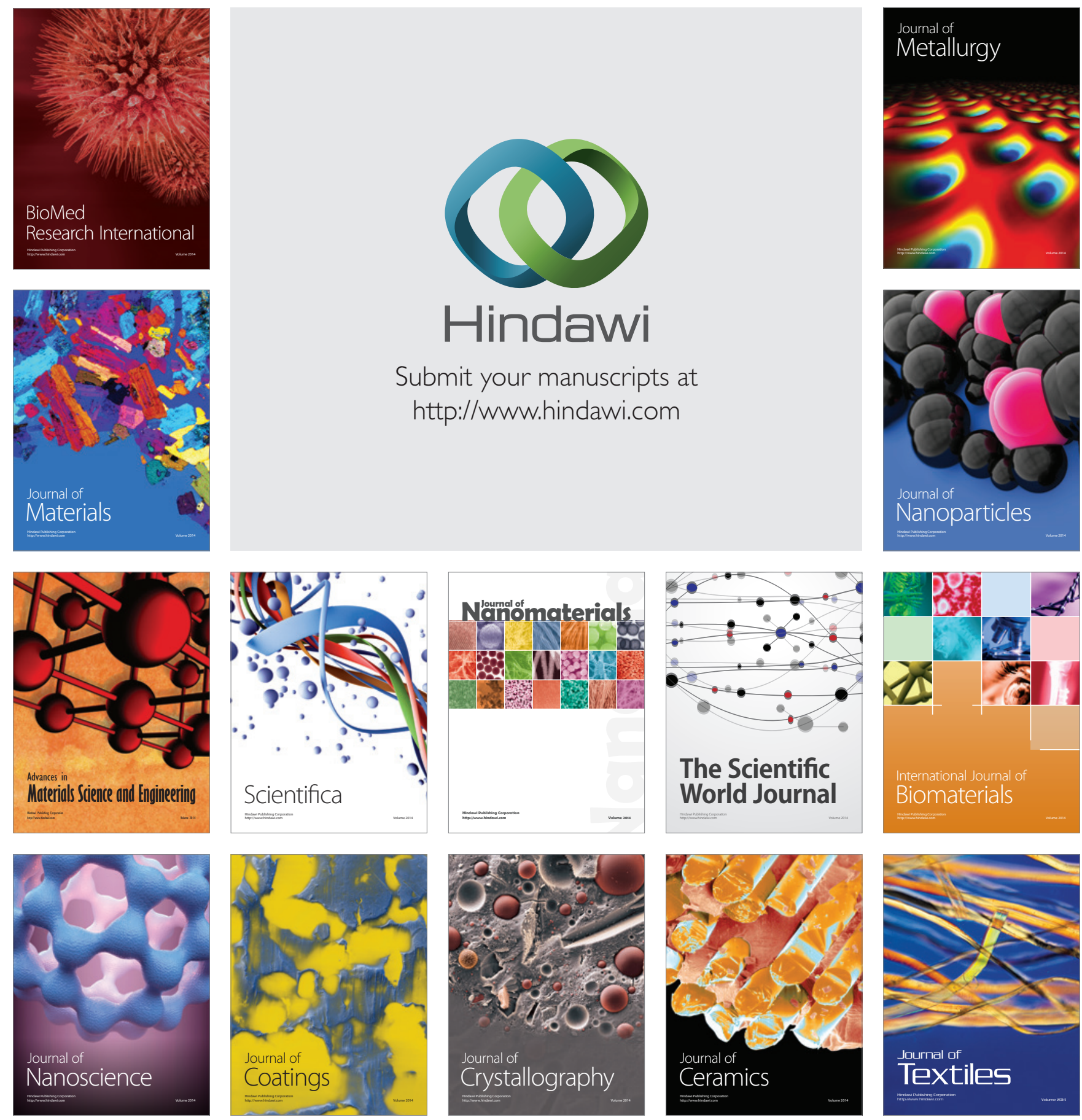